\title{
Pobreza extrema e seus tríplices fundamentos: profundidade, persistência e multiplicidade
}

\author{
Izete Pengo Bagolin ${ }^{1}$ \\ Rodrigo Peres de Ávila² \\ Flavio Vasconcellos Comim ${ }^{3}$
}

Resumo: O objetivo deste artigo é contribuir com o debate conceitual sobre pobreza extrema e propor a incorporação de novos elementos para a compreensão desse complexo fenômeno. A inexistência de clareza sobre o que é e como se mede pobreza extrema é um fato que continua a desafiar os pesquisadores. Além disso, e apesar dos avanços, as contribuições disponíveis, na sua maioria, não contemplam o processo conhecido como razão pública. Este artigo apresenta um diferencial neste aspecto, combinando elementos do debate corrente na literatura com resultados de uma pesquisa de campo que, dentre outras inovações, definiu as dimensões da pobreza a serem investigas ouvindo os pobres. Os resultados mostram que pobreza extrema está muito longe de ser apenas uma questão de intensidade na privação do acesso a recursos para sobrevivência. A pobreza extrema congrega características de intensidade, persistência e multiplicidade. Os resultados econométricos evidenciaram que o capital social, renda e escolaridade são as dimensões mais importantes para caracterizar os pobres extremos.

Palavras-chave: pobreza extrema; capacitações; multidimensionalidade.

JEL: I32; I39.

\footnotetext{
1 Doutora em Economia pela Universidade Federal do Rio Grande do Sul (UFRGS). Professora adjunta da Pontifícia Universidade Católica do Rio Grande do Sul (PUC/RS). E-mail: izete.bagolin@pucrs.br

2 Doutorado em Economia na Universidade Federal do Rio Grande do Sul (UFRGS). Professor assistente da Universidade Federal do Rio Grande (FURG). E-mail: rodrigopavila@gmail.com

3 Doutor em Economia pela University of Cambridge (Inglaterra). Professor adjunto da Universidade Federal do Rio Grande do Sul (UFRGS). E-mail: fvc1001@cam.ac.uk
} 


\title{
Extreme poverty and its triple foundation: depth, persistence and multiplicity
}

\begin{abstract}
The paper aims to contribute to the discussion on extreme poverty conceptualization and measurement of its dimensions and manifestations. It is widely known that there is no agreement about the conceptualization of extreme poverty. The literature goes from the traditional measures which are based on lack of income to the more sophisticated definitions using deprivation of basic capabilities and fundamental human rights. However, the majority of the concepts available in the literature are based on ideas and knowledge of the researchers and experts without any participation from the poor and extremely poor people. Our contribution in this paper is to put together the evolving debate from the literature and the results from a survey carried out in Porto Alegre (Brazil) during 2007. The results from the two stage questionnaire shows that that extreme poverty is far more complex than the lack of basic resources to survive, and the majority of poor people in Porto Alegre faced some of the extreme poverty characteristics during their life. The econometric results point out social capital, income and schooling as important variables to explain extreme poverty.
\end{abstract}

Key-words: extreme poverty, capability, multidimensionality.

JEL: I32; I39.

\section{Introdução}

No geral, quando se fala de pobreza extrema naturalmente vem a tona o aspecto de profundidade da pobreza, que por vezes é também chamada de miséria, indigência ou privação total de recursos. Internacionalmente, o primeiro dos oito objetivos de desenvolvimento do milênio, volta-se ao combate da pobreza extrema. Para isso, esforços consideráveis têm sido feitos pelos governos e demais instituições mundiais e de países em desenvolvimento. A meta que está sendo perseguida é reduzir, e na seqüência erradicar, o número de pessoas que vivem com menos de um dólar por dia. Já existem países e regiões que obtiveram sucesso considerável no processo de atingir essa meta. Para o Brasil, um estudo do IPEA publicado em 2009, prevê que a pobreza extrema será erradicada 2016 (IPEA, 2010). Mas sem pessoas com menos de um dólar por dia, teremos erradicado a pobreza extrema?

Que a pobreza é um fenômeno multidimensional, complexo e diverso já não existe mais dúvida na literatura. Sendo isso verdade, a pobreza extrema não seria um fenômeno ainda mais complexo e de difícil definição e enfrentamento? É razoável considerar pobreza extrema apenas como uma questão de intensidade? Ou teria esse fenômeno a ver também com os aspectos de cronicidade, multiplicidade, oportunidades e liberdades no ponto de partida? O que as pessoas que vivem em situação de pobreza consideram como sendo pobre extremo? 
Na literatura da área não existe consenso sobre como conceituar e muito menos sobre como mensurar a pobreza extrema. Instituições internacionais que são centrais no debate e formulação de estratégias para o combate da pobreza, como por exemplo, as Nações Unidas trabalham com o indicador que considera em pobreza extrema aquelas pessoas que recebem menos de um dólar por dia.

Apesar da inexistência de clareza ou consenso, desde a década de 1990, vem sendo aprofundado um debate sobre o que é pobreza extrema. As assembléias gerais da ONU sobre direitos humanos têm discutido esse tema. Apesar disso, os pesquisadores, que são considerados experts no tema não conseguiram chegar a uma definição consensual. A cada ano são apresentadas novas tentativas de desvendar esse complexo fenômeno. Existem várias definições na literatura internacional, desde as mais convencionais e unidimensionais que tomam como base a renda até as multidimensionais que enfatizam aspectos tais, como os direitos fundamentais, as necessidades, as capacitações, etc. A literatura e as medidas utilizadas no Brasil centram-se, fundamentalmente, nas definições unidimensionais, que consideram pobre extremo aquele indivíduo que se encontra abaixo das chamadas linhas de indigência.

Na seção dois são apresentadas as principais definições que mostram o processo de aprofundamento no debate internacional que faz com que as definições se tornem mais completas e, por conseqüência, também mais complexas. Apesar disso, todas as definições apresentadas ainda estão baseadas na percepção dos pesquisadores, sem considerar o que as pessoas que vivem em condição de pobreza entendem como sendo pobreza extrema. Além disso, o debate parece estar desconectado do debate mais geral sobre o conceito de pobreza e, por conseqüência não ter evoluído para o campo da mensuração dessa manifestação da pobreza.

No que tange ao primeiro aspecto, o da ligação com a conceituação da pobreza, sabe-se que a pobreza pode ser vista como relativa ou absoluta e que estas definições estão intimamente ligadas aos aspectos da intensidade, incidência e permanência (cronicidade) da pobreza. Esse debate, porém, ainda não contempla a pobreza extrema. No que se refere à mensuração, usualmente considera-se apenas o aspecto da intensidade e, ainda assim, de um ponto de vista unidimensional tomando-se a renda como proxy. De uma forma geral, o conceito de pobreza extrema tem sido utilizado de forma a retratar uma condição de privação extrema em termos dos recursos que as pessoas têm ou não acesso.

Neste sentido pode-se questionar se a combinação de aspectos que considerem as privações persistentes (cronicidade da pobreza), profundas (intensidade da pobreza) e múltiplas (multidimensionalidade da pobreza) não seriam capazes de melhor definir o que é ser pobre extremo? 
Esse artigo busca contribuir com o debate a partir de dois pontos, quais sejam: Contribuir com o processo de conceituação da pobreza extrema a partir de um processo de razão pública, onde os pobres são participantes no processo de identificação das dimensões que constituem pobreza extrema. E, a partir de um trabalho de campo, onde foram entrevistadas mais de 10 mil pessoas, busca-se identificar quais dentre os pobres, são também, extremamente pobres e quais as dimensões que caracterizam essa condição. Assim, os objetivos deste artigo são: 1) contribuir com o aprimoramento do conceito de pobreza extrema; 2) apresentar os elementos identificados pelas pessoas pobres para a definição da pobreza extrema; 3) testar as inter-relações entre as diferentes dimensões da pobreza para melhor compreender as causas e efeitos da pobreza extrema. Para atingir os objetivos propostos parte-se do debate existente na literatura sobre pobreza buscando apresentar as principais contribuições sobre os conceitos de pobreza absoluta e relativa, dando ênfase a intensidade, incidência e cronicidade.

O artigo segue a seguinte estrutura. Além desta introdução, na seção dois é apresentada uma breve revisão sobre intensidade, incidência e cronicidade e a contribuição da abordagem das capacitações. A seguir, na seção 3 são apresentados os procedimentos metodológicos e os principais resultados da pesquisa. Na seção 4 são estimadas as relações entre pobreza extrema, variáveis de controle e dimensões da pobreza. Por fim, são apresentadas as considerações finais.

\section{Um breve panorama sobre o debate envolvendo pobreza extrema e suas manifestações}

Quando se menciona o termo pobreza, é necessário ter claro quais os fundamentos conceituais que estão sendo utilizados. Ou seja, é necessário ter claro se estaremos nos referindo a pobreza absoluta ou relativa e, ainda, se é pobreza crônica ou transiente, unidimensional ou multidimensional, por exemplo.

Autores como Hulme e Shepherd (2003; 2005); Mckay e Lawson (2003) e Yaqub (2000; 2003), por exemplo, dedicam-se mais especificamente a questão da cronicidade da pobreza. A compreensão do termo pobreza crônica passa pelo entendimento do estado e das condições de privação que um indivíduo experimenta ao longo de sua vida. Segundo Hulme \& Shepherd (2003), pobreza crônica pode ser observada quando um indivíduo experimenta privações significativas de suas capacitações por um período ininterrupto de pelo menos cinco anos. Da mesma forma, a cronicidade da pobreza pode ser definida pela extensa duração em que um indivíduo sofre privações em suas capacitações (Mckay e Lawson, 2003). 
No entanto, o estado de pobreza crônica pode estar relacionado a vários aspectos, como os fatores intergeracionais, que procuram explicar a cronicidade da pobreza a partir de aspectos passados de geração para geração ou a condição dos pais pode influenciar a condição em que os filhos irão encontrar-se. De acordo com Hulme e Shepherd (2003) quanto mais tempo um indivíduo permanece em estado de pobreza, maior será sua probabilidade de permanecer pobre durante a vida toda, podendo ainda transmitir essa condição às gerações subseqüentes. Nestes termos, em sua dimensão mais estrita, a cronicidade da pobreza está ligada com elementos temporais que estão seguramente refletidos em questões intergeracionais. Pode-se relacionar a isso o debate de Sen sobre o processo de adaptação de preferências e/ou expectativas. Abordando a questão, apesar de não estar necessariamente discutindo a conceituação de pobreza crônica, a contribuição de Sen auxilia no entendimento de questões relacionadas com a não ruptura ou "acomodação" das pessoas a condições de privação.

Para Mckay e Lawson (2003), as principais definições de pobreza crônica estão relacionadas à sua duração e a sua natureza multidimensional. Clark e Hulme (2005) corroboram essa visão afirmando que o debate sobre pobreza evoluiu de forma significava e tornou evidente a importância da multidimensionalidade, da profundidade e da persistência da pobreza - que são, na visão destes autores, os componentes da pobreza crônica.

A distinção entre pobreza crônica e pobreza transitória é geralmente ligada à conceitualização de pobreza na dimensão monetária. Isso, em grande parte, está relacionado ao fato de que a natureza estocástica do processo de geração de renda é bem reconhecido na economia, destacando à hipótese de Renda Permanente de Friedman, (Friedman, 1957). Neste contexto, o consumo é usado como o indicador preferido de bem-estar em várias aplicações em países em desenvolvimento por acreditar que isso é um reflexo melhor para rendas permanentes ou de longo prazo. Neste sentido, o baixo consumo visto como um reflexo da incapacidade crônica para gerar rendas suficientes para abandonar a pobreza, mesmo que famílias possam temporariamente escapar da pobreza de renda (Gunter e Klasen, 2007).

Ao mesmo tempo, essa ênfase em rendas na avaliação de pobreza crônica e vulnerabilidade têm claras limitações e problemas. Conforme, Hulme e McKay (2005), a renda (ou consumo) é um indicador inadequado de bem-estar. Se nós conceituamos bem-estar de uma perspectiva de capacitações, renda nada mais é do que um (e para algumas capacitações um pobre) meio para gerar capacitações tais como a habilidade para ser saudável, bem-educado, integrado, vestido e outras capacitações do gênero, nem mesmo rendas iguais traduzem em capacitações iguais para indivíduos diferentes, por causa da heterogeneidade das pessoas para traduzir rendas em bem-estar. É preferível então estudar resultados de bem-estar diretamente, ao invés de estudar um input específico de bem-estar. Porém, existiram algumas tentativas para 
integrar as introspecções da análise estática de dimensões não-renda de bem-estar em um cenário dinâmico e assim investigar a pobreza crônica e vulnerabilidade de um ponto de vista não-renda (Sen, 1998 apud Gunter e Klasen, 2007).

A cronicidade da pobreza pode ainda ser analisada em termos das privações absoluta ou relativa. A maioria dos trabalhos aborda a pobreza crônica absoluta. No entanto, Yaqub (2003) apud Ribas et al. (2005) argumentam que, referente ao indivíduo que está sempre no mesmo quintil de distribuição de renda, a pobreza crônica relativa seria de tão difícil saída, ou mais, quando comparada a absoluta. Nesta mesma linha, Sen (1981) expõe que a privação relativa em termos de bens, renda ou recursos estaria relacionada à privação absoluta em termos de capacitações das pessoas.

No foco das características dos indivíduos e das famílias em situação de pobreza crônica, esta consideração não permite apenas verificar como a cronicidade da pobreza difere da pobreza transitória, mas talvez mais importante seja considerar políticas com a finalidade de combater a pobreza crônica, baseadas sobre uma sólida compreensão de quais são os determinantes dessa situação. As características geralmente associadas com pobreza crônica incluem uma situação desvantajosa com respeito a um ou a mais dos seguintes aspectos: capital humano; composição demográfica; posição; recursos físicos; e, categoria ocupacional, entre outras. Isto corresponde às expectativas prévias e também à compreensão baseada na pobreza crônica desenvolvida por Gaiha (1992), em que a pobreza crônica reflete níveis baixos de ativos e/ou de retornos persistente baixos dos ativos (Mckay e Lawson, 2003).

Sen (1997) usa as capacitações com o objetivo para demarcar o espaço dentro do qual a avaliação da qualidade de vida se dá. Assim, ao invés de questionar sobre a quantidade de recursos que um indivíduo está apto a dominar ou sobre as suas satisfações, Sen (1985a, 1985b, 1985c, 1992, 1997, 1999) questiona sobre o que as pessoas são realmente capazes de fazer ou de ser (quão bem estes indivíduos estão indo), ou seja, questiona dentro do espaço das capacitações.

Já, Nussbaum (2000) propõe mover-se além do uso meramente comparativo das capacitações para empregá-las em um sentido mais operacional/prático, apresentando-as como um fundamento para elaboração de princípios políticos básicos, que deveriam ser considerados na elaboração de princípios constitucionais. Desse modo, a Abordagem das Capacitações deveria direcionar as preocupações para o exame das condições sociais e materiais da vida humana.

Nesse sentido, a Abordagem das Capacitações é utilizada por Nussbaum (2000, p. 5) como uma base filosófica para o estabelecimento de uma "lista de princípios constitucionais básicos que devem ser respeitados e implementados 
pelos governos de todas as nações, como um mínimo essencial que respeita os requerimentos da dignidade humana". Em função disso, Nussbaum (2000) defende a existência e a possibilidade de definição de uma lista de capacitações humanas centrais, que pode ser entendida como um nível a partir do qual a pessoa passa a ser o que se poderia chamar de "verdadeiramente humano" ou capaz de exercer a sua condição de agente. Em contrapartida, as situações nas quais as pessoas se encontram abaixo deste nível em uma das áreas centrais do funcionamento humano, deveriam ser consideradas como situações trágicas e injustas. Ou seja, em um sentido extremo, pode-se julgar que a ausência de capacitação para funcionar pode ser suficientemente forte que não permite que a pessoa se torne um ser humano, ou seja, pode ser considerada extremamente pobre.

Analisando a literatura, é possível encontrar um conjunto de definições para pobreza extrema. Estas definições, apesar de possuírem aspectos comuns, divergem em vários aspectos e confirmam a inexistência de uma definição consensual ou padrão. A seguir são apresentadas algumas das definições encontradas na literatura. Como é possível perceber existem definições contemplando os aspectos da multidimensionalidades, da cronicidade e da profundidade da pobreza, como por exemplo a que foi apresentada no relatório da conferência sobre direitos humanos de 1996 que afirma:

Poverty becomes extreme poverty when it "simultaneously affects several aspects of people's lives, when it is prolonged and when it severely compromises people's chances of regaining their rights and of reassuming their responsibilities in the foreseeable future. (E/CN.4/Sub.2/1996/13)

Em 2000 o comitê de direitos humanos define pobreza em seu relatório enfatizando apenas aspectos de exclusão e dignidade, como é possível perceber na definição a seguir.

...extreme poverty and exclusion from society constituted a violation of human dignity and called for urgent national and international action to eliminate them HRC Res. 55/106/2000.

Em 2004 o mesmo comitê define pobreza extrema como sendo um processo de violação de direitos humanos fundamentais e liberdades.

extreme poverty places men, women, children and entire population groups in a situation involving the violation of fundamental rights and freedoms, in the industrialized countries as much as in the developing countries, that it may, in some situations, constitute a threat to the right to life, and that its immediate alleviation and eventual eradication must remain a high priority for the international community. (HRC, 2004). 
...the extreme poverty should be defined in terms of a composite of income poverty, human development poverty and social exclusion, and that such a definition would incorporate in a comprehensive manner the notions of the lack of basic security, capability deprivation and social exclusion and would build on the multidimensionality of the concept and take account of the interdependence of different constitutive elements. (HRC, 2005:53)

...in recent year, international organizations and specialized agencies have reached a consensus that extreme poverty is a denial of fundamental human rights, that it prevents the effective realization of human rights, and that human rights violations and situations of extreme poverty are closely interlinked. (HRC, 2006: 5)

In my view, it is simplest to think of poverty as failure of 'basic capabilities' and then to define extreme poverty as severe failure of basic capabilities. In this case, there will be two thresholds for each basic capability - one at higher level to define poverty and another at a lower level to define extreme poverty. (HRC, 2007: 2)

Na literatura brasileira, bem como nas instituições governamentais que atuam no combate a pobreza o significado da pobreza extrema é pouco debatido e não existe um conceito claro do que ela representa. A métrica que tem sido utilizada para orientar as medidas de política econômica é a linha de pobreza tradicional de 1 ou 2 dólares ou linhas vinculadas ao salários mínimo e frações deste referindo-se ao que no Brasil de chama de linha de indigência. São consideradas extremamente pobres (ou indigentes), aquelas pessoas que estiverem abaixo de um determinado limite. Exemplo disso é o que está na definição do IBGE.

... no Brasil, a metodologia oficial usa como referência o Salário Mínimo, isto é, 1/4 do salário mínimo familiar per capita e 1/2 do salário mínimo familiar per capita, limites abaixo dos quais se define uma família extremamente pobre (indigente) e pobre, respectivamente.

A partir deste panorama e das muitas questões a serem respondidas a próxima seção destina-se a mostrar os procedimentos metodológicos que foram utilizados para construir o índice de pobreza extrema para Porto Alegre. 


\section{Procedimentos Metodológicos para coleta dos dados e construção de um indicador de pobreza extrema}

\subsection{Coleta de dados}

Os dados utilizados neste artigo são provenientes de uma pesquisa inédita realizada em 2007 na cidade de Porto Alegre. Essa pesquisa possui particularidades que é importante destacar, quais sejam: (1) as questões utilizadas no questionário foram construídas junto com os pobres da cidade, sendo uma forma de dar voz aos mesmos. Seguindo o que a literatura chama de processo de razão pública; (2) a pesquisa foi realizada em duas etapas (foram plicados dois questionários onde no primeiro buscou-se identificar como os pobres vêem sua própria pobreza, quais são suas dimensões e qual a importância de cada dimensão; (3) o segundo questionário foi construído com base nas respostas obtidas no primeiro; (4) a aplicação dos questionários foi feita após a capacitação de pessoas das próprias comunidades, visando também um processo de empoderamento e conscientização da população sobre suas próprias privações; (5) As questões do questionário 2 buscaram identificar intensidade das privações no espaço das capacitações (ou fins do desenvolvimento) e não apenas sobre a posse ou não de recursos (meios).

Os questionários foram aplicados em amostras da população pobre de Porto Alegre com aproximadamente 8.00o pessoas entrevistadas na primeira fase (consulta) e mais de 10.000 mil pessoas na segunda fase (coleta dos dados propriamente dita). Todas as regiões da cidade estiveram representadas. A seguir são apresentados os procedimentos para construção do indicador de pobreza extrema.

\subsection{Indicador de pobreza extrema}

A pobreza extrema é destacada aqui como uma categoria distinta de pobreza. Isso porque a natureza dos problemas enfrentados pelas pessoas é diferente no que tange a intensidade, dimensões das privações e, também quanto a persistência destas privações. Conforme exposto anteriormente, o conceito de pobreza extrema não é claro na literatura. No entanto, nesta seção, apresenta-se um indicador específico de pobreza extrema - índice de pobreza extrema (IPE), que foi idealizado a partir dos indicativos das próprias pessoas pobres sem, no entanto, sucumbir aos limites dos indicadores subjetivos. Busca-se com este indicador, identificar dentre as pessoas já consideradas pobres, quais sofrem as privações mais severas ou extremas. O indicador foi construído para variar entre $\mathrm{o}$ e 1 , sendo consideradas extremamente pobres as pessoas com IPE $>0.33$. Vale ressaltar que esse parâmetro é arbitrário e serve apenas para que se possa estimar o número de pessoas nessa situação e suscitar o debate em torno do conceito de pobreza extrema. Assim, não se 
deve interpretar que as pessoas com IPE < 0.33 não estejam sujeitas a algum nível de pobreza extrema. Como o IPE possui várias dimensões, pode-se encontrar um indivíduo que não seja extremamente pobre na média, mas sofra privações de alguns funcionamentos importantes. Rigorosamente, só pode ser considerado efetivamente como "não pobre extremo" aquele indivíduo que tiver IPE $=0$. Deste modo, ressalta-se que se o indivíduo tem algum grau de pobreza extrema. A interpretação do IPE segue a mesma racionalidade do IPH, onde quanto mais próximo de 1 o valor estimado, mais pobre extremo a pessoa é, e quão mais perto de zero menor a pobreza.

O IPE é composto por seis indicadores ou dimensões, que durante o processo de consulta foram identificadas como sendo as dimensões ou características que revelam (ou diferenciam) a condição de pobreza extrema. Estas características foram mensuradas na segunda etapa buscando identificar sua presença e intensidade das privações na vida das pessoas. Cada uma destas dimensões (ou variáveis) é detalhada e justificada a seguir.

i) Variável "Esmola": questão que buscou estimar se o entrevistado já havia pedido esmola na rua, qual a periodicidade com que isto acontecia e se isto aconteceu por períodos longos de tempo. Nesta variável, usou-se a informação do entrevistado sobre o "tempo de esmola" para diferenciar aqueles que esmolam a pouco tempo daqueles que o fazem de longa data. A persistência da pessoa nesta condição revela a face da cronicidade associada a intensidade. Assim, quanto mais perto de 1 estiver a variável, mais tempo faz que o indivíduo encontra-se nessa situação . Caso o indivíduo nunca tenha pedido esmola, o indicador é igual a zero. Veja que algumas pessoas muito pobres não necessariamente podem ter passado pela experiência de pedir esmola. Esta dimensão, assim como as demais incluídas nesse índice, busca ser específica da condição de pobreza extrema por ter sido uma resposta muito freqüente durante a consulta que ser miserável ou pobre extremo significa ter que pedir esmola.

ii) Variável "Humilhação": questão que buscou averiguar se o entrevistado alguma vez já sofreu algum tipo de constrangimento em função de sua condição de pobreza. Quando o entrevistado declarou já ter sido humilhado, tem-se o indicador dessa questão igual a 1; por conseqüência, quando declarou nunca ter sido humilhado, tem-se o indicador igual a zero. Deve-se enfatizar aqui a relação associativa perguntada entre humilhação e pobreza. Outros tipos de humilhação, não-associados à pobreza, não foram considerados. Essa variável foi considerada importante pois os pobres referiram que uma face da pobreza é o fato das pessoas não se sentirem "gente", de serem tratadas com desprezo ou com injustiça pelo fato de serem "visivelmente" pobres. 
iii) Variável "Moradia": questão formulada com o intuito de medir se os indivíduos possuem local onde possam desfrutar de uma noite de sono. Caso o indivíduo tenha declarado que alguma vez dormiu na rua por não ter para onde ir, tem-se o indicador igual a 1; caso tenha respondido negativamente, tem-se que seu indicador é igual a zero. Foi surpreendentemente elevado o número de pessoas pobres que responderam que ser miserável é não ter para onde ir. A pobreza extrema aqui estava sendo associada a viver na rua.

iv) Variável "Frio": questão com o propósito de captar o número de pessoas entrevistadas que já passaram frio por não possuir roupas adequadas ou por estarem em local desprotegido. Se o entrevistado declarou que já passou frio por um desses motivos, tem-se o seu indicador igual a 1; caso contrário tem-se o indicador igual a zero. Reconhece-se que essa variável tem, a priori um poder descriminatório mais baixo, por ser mais comum a experiência de passar frio entre os indivíduos mais pobres mas sua inclusão justifica-se pois as pessoas manifestaram que pobreza extrema é não ter o que vestir.

v) Variável "Fome": questão formulada para estimar se o entrevistado alguma vez no último mês ficou um dia inteiro sem comer por não possuir dinheiro para comprar comida para si ou para os seus filhos. Os indivíduos que responderam positivamente tiveram seu indicador igualado a 1 , da mesma forma que os indivíduos que declararam não ter passado fome, no último mês, tiveram seu indicador igualado a zero. Apesar desta variável ser, de certa forma, similar a variável 1, ela busca captar ênfase diferente que foi dada na caracterização da pobreza extrema. Esta variável centra-se no aspecto alimentação e visa captar o aspecto "não ter o que comer", que tem mais a ver com a intensidade, enquanto que a variável esmola é mais abrangente captando também persistência e/ou cronicidade.

vi) Variável "Confiança": questão que se propôs a medir em quantas pessoas cada indivíduo entrevistado confia. Acredita-se que esta questão é importante no âmbito do estudo da miserabilidade ou extrema pobreza, visto que no questionário aplicado anteriormente boa parte dos entrevistados referiram que é miserável aquele que não tem ninguém que possa ajudá-lo nos momentos de dificuldade. Assim, caso o entrevistado tenha assinalado que não confia em ninguém, teve seu indicador considerado igual a 1; caso tenha assinalado que confia em uma ou mais pessoas, seu indicador foi considerado igual a zero.A pergunta buscava identificar com quem efetivamente a pessoa pode contar nos momentos de dificuldade.

Não obstante, duas idéias centrais merecem ser reforçadas. A primeira é de que cada um dos seis componentes do IPE permite a estimação para cada indivíduo entrevistado, resultando obviamente em um índice geral para cada pessoa. A segunda idéia central é que todas as seis variáveis que compõem o IPE têm a mesma importância, ou seja, cada uma dessas variáveis tem peso $1 / 6$ na formulação final. Desta forma, pode-se escrever o Indicador de Pobreza Extrema do indivíduo i de acordo com a seguinte expressão: 


$$
I P E_{i}=\left(\frac{V_{\text {esmola }}+V_{\text {humilhação }}+V_{\text {moradia }}+V_{\text {frio }}+V_{\text {fome }}+V_{\text {confiança }}}{6}\right)
$$

Embora seja um indicador calculado individualmente, salienta-se que não há qualquer restrição matemática ou metodológica à agregação dos indicadores e obtenção de valores médios para grupos ou localidades diferentes. Nesse sentido, além dos cálculos para Porto Alegre, optou-se por estimar um índice equivalente por gênero e também para cada uma das 16 regiões do OP. Em outras palavras, para calcular o IPE para a cidade como um todo ou para alguma região, basta calcular a média do índice entre todos os moradores do local de interesse. Da mesma forma, para estimar o IPE por gênero, basta calcular separadamente o índice médio dos indivíduos dos sexos masculino e feminino.

A vantagem desse procedimento é a possibilidade de investigação sobre as diferenças no padrão de distribuição da miserabilidade entre localidades e diferentes grupos de interesse.

\subsection{Resultados da pesquisa}

Para o cálculo do IPE foram utilizadas as respostas 8998 pessoas. O resultado mais expressivo é que, dentre este total, tem-se 3087 possuem IPE superior a 0,30 o que as classifica como extremamente pobres. Isso corresponde a 34,3\% das pessoas da amostra.. Em situação oposta, tem-se 3310 indivíduos com IPE $=0$. Tais pessoas, cuja proporção é de $36,8 \%$, apesar de serem pobres na dimensão renda, não podem ser consideradas extremamente pobres, de acordo com o critério e o conceito utilizado nessa pesquisa. Existe, ainda, o grupo das pessoas com grau de pobreza extrema diferente de zero, mas que de acordo com o parâmetro considerado não estariam entre os que enfrentam situação de pobreza extrema. Em outras palavras, são pessoas com baixas privações características da pobreza extrema.

Na figura 1, a seguir, estão os valores da pobreza extrema em Porto Alegre para cada uma das dimensões consideradas. É possível perceber que a dimensão que apresenta o valor mais elevado de privação extrema é a variável frio, que como vimos na descrição acima, capta as privações nas condições proteger-se das adversidades climáticas e não passar frio por falta ou insuficiência de roupa e proteção adequada. Esse resultado é importante e revelador da importância das particularidades locais e regionais que caracterizam a pobreza pois mostra um alto grau de privação numa dimensão que possivelmente não apareceria nas outras regiões do país e que no Sul pode ser fatal. Outro aspecto as ser destacado refere-se a dimensão humilhação. 
Essa dimensão mostra que é elevado o número de pessoas que são desprezadas pelo restante da sociedade por serem visivelmente pobres e, por isso, não merecedoras de respeito e tratamento digno e igualitário.

Por outro lago, a característica que no senso comum do Brasileiro é a principal característica do pobre extremo - aquele que pede esmola - apresentou valor inferior as demais dimensões destacadas pelo pobres como características importantes da pobreza extrema. Isso mostra que mesmo pessoas que não estão pedindo esmola vivem em condição considerada de miserabilidade ou pobreza extrema.

FIGURA 1. INTENSIDADE DAS PRIVAÇÕES NAS DIMENSÕES DO ÍNDICE DE POBREZA EXTREMA (IPE) DA CIDADE DE PORTO ALEGRE

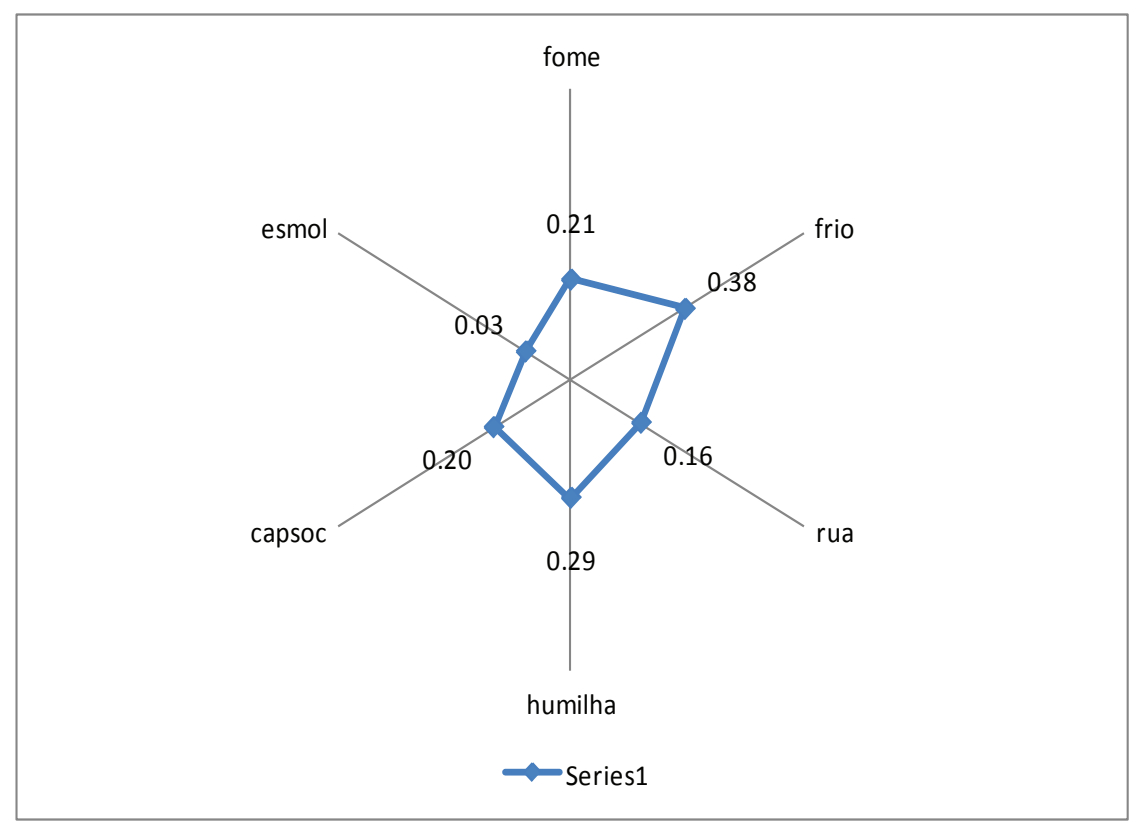

FONTE: elaboração dos autores.

Imagine por um instante que a cidade de Porto Alegre fosse representada por apenas um indivíduo dessa amostra, esse agente representativo teria $\mathrm{IPE}=0.21$. Isto indica que a cidade estaria no grupo que não marcado pela pobreza extrema, mas também não está livre de alguma de suas características, mesmo que relativamente moderada. Este raciocínio pode ser bastante útil se quisermos comparar sinteticamente cada região da cidade com a média municipal. 


\subsubsection{Ranking comparativo entre regiões (IPE)}

Analisando o índice por região da cidade percebe-se que existe heterogeneidade na incidência da pobreza extrema entre as regiões de Porto Alegre. Neste sentido, a tabela 1, resume as informações relativas ao Indicador de Pobreza Extrema por região.

Percebe-se que dez regiões se encontram em situação relativamente melhor do que a cidade como um todo. Tal fato sugere que as seis regiões com desempenho pior do que a média acumulam um percentual elevado da amostra. A região Centro foi a que obteve pior desempenho, classificada como número um no ranking da pobreza extrema. As regiões Cristal, Restinga e Nordeste também apresentaram IPE superior às demais localidades.

TABELA 1. REGIÕES NO INDICADOR DE POBREZA EXTREMA

\begin{tabular}{|c|c|c|c|}
\hline Regiões & $\begin{array}{c}\text { \% de Pobres } \\
\text { Extremos }\end{array}$ & IPE médio & $\begin{array}{l}\text { Ranking } \\
\text { IPE médio }\end{array}$ \\
\hline Humaitá Navegantes Ilhas & $36,0 \%$ & 0,2039 & 8 \\
\hline Noroeste & $38,4 \%$ & 0,1938 & 11 \\
\hline Leste & $30,1 \%$ & 0,1976 & 9 \\
\hline Lomba do Pinheiro & $30,9 \%$ & 0,1752 & 15 \\
\hline Norte & $27,7 \%$ & 0,1839 & 13 \\
\hline Nordeste & $44,8 \%$ & 0,2668 & 4 \\
\hline Partenon & $36,6 \%$ & 0,1935 & 12 \\
\hline Restinga & $45,4 \%$ & 0,2669 & 3 \\
\hline Glória & $25,0 \%$ & 0,1627 & 16 \\
\hline Cruzeiro & $30,9 \%$ & 0,2056 & 7 \\
\hline Cristal & $47,5 \%$ & 0,2759 & 2 \\
\hline Centro-Sul & $27,3 \%$ & 0,1807 & 14 \\
\hline Extremo-Sul & $30,3 \%$ & 0,1964 & 10 \\
\hline Eixo-Baltazar & $36,8 \%$ & 0,2294 & 5 \\
\hline Sul & $32,9 \%$ & 0,2246 & 6 \\
\hline Centro & $49,1 \%$ & 0,3251 & 1 \\
\hline Porto Alegre & $34,3 \%$ & 0,2131 & - \\
\hline
\end{tabular}

FONTE: elaborado pelos autores. 
No outro extremo, temos as regiões com desempenho relativamente satisfatório, exemplo disso é a região da Glória, que tem "apenas" $25 \%$ de seus entrevistados em situação de miserabilidade. As regiões Lomba do Pinheiro e Centro-Sul também se destacaram relativamente nesse sentido.

Os valores e percentuais apresentados podem, num primeiro momento, parecer pouco expressivos. No entanto, quando comparados aos dados convencionais sobre pobreza extrema, como, por exemplo, os dados do IPEA, que mostram que no Rio Grande o Sul, apenas. $6 \%$ da população vive em condições de pobreza extrema os resultados dessa pesquisa revelam-se a complexidade e importância de uma conceituação e mensuração correta do fenômeno em estudo. Os resultados aqui apresentados reforçam a idéia de que a renda é uma dimensão importante e necessária tanto na mensuração quanto no combate a pobreza, mas não se constitui em elemento suficiente.

\section{Análise do impacto de cada dimensão da pobreza na determinação da pobreza extrema}

Buscando uma melhor compreensão da relação entre pobreza extrema e as características das pessoas entrevistadas, estimou-se um modelo econométrico simples, com a seguinte especificação:

$$
I P E=f\left(X_{i}, Y_{i}, E_{i}, C S_{i}\right),
$$

Onde:

$I P E=$ índice de pobreza extrema

$X_{i}=$ conjunto de características individuais de cada pessoa (nível de escolaridade, idade, gênero, Região da cidade onde mora);

$Y_{i}=$ faixa de rendimento em faixa de salários mínimos na qual a pessoa se encontra;

$E_{i}=$ variável emprego. Assume valor um se a pessoa nunca teve emprego formal, e zero que a pessoa já trabalhou ou está trabalhando com carteira assinada;

CS = variável que mensura o "grau" de capital social da pessoa pelo número de pessoas em que ela confia. Assume o valor 1 se a pessoa não confia em ninguém e zero se a pessoa confia em uma ou mais pessoas. 
Foram estimadas diversas regressões, mas optou-se por apresentar apenas a que apresentou ajuste adequado de acordo com os critérios de Akaike e de Schwarz. Corrigiu-se a heteroscedasticidade pelo procedimento de White. A regressão tem $\mathrm{R} 2=0,3878$ e o teste $F$ (conjunto) mostrou-se significativo a $1 \%$. São indícios de que o modelo está bem ajustado. Na tabela 2, são apresentados os resultados das estimações.

Os resultados indicam que a variável mais importante para a explicação do nível de pobreza extrema em Porto Alegre é o Indicador de Capital Social, sendo seu coeficiente sensivelmente superior aos das demais variáveis explicativas. Deste modo, pessoas que declararam ter confiança nos demais, ter a quem recorrer em momentos de dificuldade, apresentaram índices de pobreza extrema relativamente baixos. Vale ressaltar que a importância do capital social se mostrou evidente durante todo o processo de pesquisa, mas sua força na explicação do fenômeno da pobreza extrema ainda assim é, em certa medida, surpreendente.

Em Ávila, Bagolin e Comim (2008), o coeficiente do mesmo indicador de capital social na regressão que buscou entender os determinantes do índice de carências multidimensional foi de-0,08557, enquanto no presente trabalho é de -0,305445. Ademais, na presente regressão, o segundo maior coeficiente estimado é de -0,160639, aproximadamente metade do impacto do capital social. São indícios de quão expressivo é o resultado obtido. 


\section{TABELA 2. COEFICIENTES ESTIMADOS}

\begin{tabular}{|c|c|c|c|}
\hline \multicolumn{4}{|c|}{ Variável Dependente: Índice Pobreza Extrema } \\
\hline Variável Explicativa & Coeficiente & $\begin{array}{c}\text { Erro } \\
\text { Padrão }\end{array}$ & Teste $t$ \\
\hline Intercepto & 0.568052 & 0.021410 & 26.53177 \\
\hline De 1 até 4 anos de Estudo & -0.048680 & 0.012750 & -3.818145 \\
\hline De 5 até 8 anos de Estudo & -0.119966 & 0.012476 & -9.616116 \\
\hline Mais de 8 anos de Estudo & -0.160639 & 0.012929 & -12.42494 \\
\hline Idade & 0.003385 & 0.000697 & 4.854585 \\
\hline$(\text { Idade })^{\wedge} 2$ & $-4.11 \mathrm{E}-05$ & $7.89 \mathrm{E}-06$ & -5.210663 \\
\hline Gênero $(*)$ & -0.005891 & 0.004486 & -1.313319 \\
\hline Região $1(*)$ & 0.008323 & 0.009922 & 0.838799 \\
\hline Região $2(*)$ & 0.014192 & 0.010550 & 1.345165 \\
\hline Região $3(*)$ & 0.007076 & 0.011124 & 0.636048 \\
\hline Região $4\left(^{*}\right)$ & 0.006897 & 0.012346 & 0.558628 \\
\hline Região 5(*) & 0.014813 & 0.008397 & 1.764184 \\
\hline Região 6 & 0.032218 & 0.013050 & 2.468757 \\
\hline Região 8 & 0.045804 & 0.010701 & 4.280215 \\
\hline Região 9 & -0.027321 & 0.008896 & -3.071334 \\
\hline Região 10 & -0.018331 & 0.009167 & -1.999626 \\
\hline Região 11(*) & 0.009094 & 0.020218 & 0.449764 \\
\hline Região 12(*) & -0.008594 & 0.012267 & -0.700620 \\
\hline Região $13\left(^{*}\right)$ & -0.008101 & 0.009863 & -0.821314 \\
\hline Região 14(*) & 0.005966 & 0.015827 & 0.376935 \\
\hline Região 15(*) & -0.026940 & 0.017527 & -1.537075 \\
\hline Região 16 & 0.022603 & 0.009582 & 2.358816 \\
\hline Renda até $1 \mathrm{SM}\left({ }^{*}\right)$ & 0.000297 & 0.007680 & 0.038673 \\
\hline Renda entre 1 e 2 SM & -0.056076 & 0.006789 & -8.259587 \\
\hline Renda entre 3 e 4 SM & -0.080567 & 0.008741 & -9.217292 \\
\hline Renda entre 4 e $5 \mathrm{SM}$ & -0.089231 & 0.014957 & -5.965965 \\
\hline Renda maior que 5 SM & -0.081060 & 0.022250 & -3.643144 \\
\hline Emprego Formal & 0.038608 & 0.004760 & 8.111019 \\
\hline Indicador de Capital Social & -0.305445 & 0.007399 & -41.28261 \\
\hline
\end{tabular}

FONTE: elaborado pelos autores.

NOTA: $\left({ }^{*}\right)$ variáveis não significativas a $5 \%$ (teste $\mathrm{t}$ ). 
Conforme esperado, as dummies de renda monetária também apresentaram forte impacto na explicação da pobreza extrema. Os resultados da tabela devem ser interpretados em relação à variável omitida, a dummy para pessoas sem rendimento declarado. Desta forma, pessoa com ganhos a partir de 1 salário mínimo experimentaram níveis de pobreza extrema menores. Um fato interessante a ser sublinhado é que o valor do coeficiente estimado não mudou sensivelmente entre os níveis de renda analisados, o que indica que mudar de patamar de renda não implica necessariamente em redução de pobreza extrema, se não for acompanhada de outras características, tal como escolarização e capital social. $\mathrm{O}$ fato da variável que representa as pessoas com ganhos de até 1 salário mínimo não ter sido significativa não chega a surpreender, tendo como provável explicação a difícil distinção entre aqueles que declararam não ter rendimento algum e aqueles que ganham muito pouco.

Ainda no âmbito do trabalho e dos rendimentos, destaca-se o fato da variável emprego formal ter se apresentado significativa, mas com sinal positivo, contrário ao esperado. A provável explicação para esse fato é que um dos componentes da pobreza extrema é a variável humilhação, que muitas vezes esteve vinculada ao emprego dos entrevistados.

Já as variáveis vinculadas ao estoque de educação formal apresentaram-se significativas e com sinal esperado. Mais do que isso, a magnitude do impacto negativo da escolarização no nível de pobreza extrema tende a ser mais forte quando os indivíduos estudam mais. Os resultados devem ser interpretados em relação à dummy omitida, que é a das pessoas sem escolaridade formal. Nota-se que o impacto da mudança de nível de escolaridade é bastante forte na redução da pobreza extrema, tendo o valor do coeficiente triplicado do primeiro ao último grupo.

As demais variáveis utilizadas no modelo tiveram impacto pequeno ou não foram significativas estatisticamente. Destaque para a variável gênero, que ao mostrar-se não significativa indica não haver, para a situação de pobreza extrema, indícios do fenômeno de feminilização da pobreza extrema em Porto Alegre. Outro fator ser observado é que as variáveis espaciais mostraram-se majoritariamente não significativas, e mesmo as que foram significativas apresentaram coeficiente pequeno. Isso indica que a pobreza extrema em Porto Alegre não está explicitamente relacionada com o espaço geográfico que as pessoas ocupam dentro da cidade. 


\section{Considerações finais}

A partir da literatura sobre o tema é possível perceber que existe um processo de aprofundamento no debate internacional no que se refere às definições. Estas vêm se tornando mais completas e, por conseqüência, também mais complexas. Apesar disso, todas as definições apresentadas estão baseadas na percepção dos pesquisadores, sem considerar o que as pessoas que vivem em condição de pobreza entendem como sendo pobreza extrema. Além disso, o debate parece estar desconectado do debate mais geral sobre o conceito de pobreza e, por conseqüência não ter evoluído para o campo da mensuração da manifestação da pobreza. A pobreza extrema continua a ser mensurada apenas como uma questão de intensidade da pobreza.

Na pesquisa desenvolvida em Porto Alegre foi possível constatar que as pessoas pobres consideram como extremamente pobres aquelas pessoas que além de sofrerem privações intensas, essas privações são persistentes e vão além da privação de recursos físicos. Constituem-se também em privações de dignidade, direitos, oportunidades. Do total de entrevistados pela pesquisa foi possível perceber que aproximadamente $64 \%$ das pessoas sofrem de algum tipo de pobreza extrema, tendo apresentado IPE diferente de zero.

A equação estimada permite afirmar que a variável que mais contribui para explicar a pobreza extrema é a variável de capital social. O que evidencia que pobreza extrema não é uma questão apenas de recursos físicos e sim de laços humanos e de confiança.

\section{Referências bibliográficas}

BECCARIA, L. \& GROISMAN, F. (2006). "Income mobility in Argentina". National Centre for Scientific and Technical Research (CONICET) and Universidad Nacional de General Sarmiento. Argentina.

CAPPELLARI, L. \& JENKINS, S. P. (2002). Modelling Low Income Transitions. Colchester: ISER, [Working Paper].

COMIM, F. et al. (2007). Pobreza Multidimensonal e Pobreza Extrema para Porto Alegre. Porto Alegre, 2007.

GAIHA, R. \& DEOLALIKAR, A. B. (1993). "Persistent, Expected and Innate Poverty: Estimates for Semi Arid Rural South India”. Cambridge Journal of Economics, vol. 17 (4), p. 409-421.

GUNTHER, I. \& KLASEN, S. (2007). Measuring Chronic Non-Income Poverty. University of Göttingen.

HULME, D. \& MCKAY, A.(2005). Identifying and Understanding Chronic Poverty: Beyond Income Measures. Manchester: Chronic Poverty Research Center. 
HULME, D. \& SHEPHERD, A. (2003). "Conceptualizing Chronic Poverty". World Development, vol. 31 (3), p. 403-423.

HULME, D. \& SHEPHERD, A. (2005). Identifying and Understanding Chronic Poverty: Beyond Monetary Measures. University of Manchester and Chronic Poverty Research Centre.

HUMAN RIGHTS COUNCIL (HRC). Sub-commission report. United Nations. General Assembly (1996, 2000, 2004, 2005, 2006, 2007).

IPEA - Instituto de Pesquisa Econômica Aplicada (2010). "Dimensão, evolução e projeção da pobreza por região e por estado no Brasil”. Comunicados IPE, n. ${ }^{\circ} 58$.

JALAN, J. \& RAVALLION, M. (2000). "Is Transient Poverty Different? Evidence for Rural China”. Journal of Development Studies, vol. 36, n. 6, p. 82-98.

LOPES, H. M. \& MACEDO, P. B. R. \& MACHADO, A. F. (2004). “Análise de Pobreza com Indicadores Multidimensionais: uma Aplicação para Brasil e Minas Gerais”. In: XIV Encontro Nacional de Estudos Populacionais, ABEP.

MACHADO, A. F. \& RIBAS, R. P. \& PENIDO, M. (2007). “Mobilidade entre estados de pobreza e inserção no mercado de trabalho: uma análise para o Brasil metropolitano em 2004”. Economia Aplicada, vol. 11 (2), p. 253-279.

MCKAY, A.; LAWSON, D. (2003). "Assessing the Extent and Nature of Chronic Poverty in Low Income Countries: Issues and Evidence". University of Nottingham, UK. World Development, vol 3, p. 425-439.

NUSSBAUM, M. (2000). Woman and Human Development: the capabilities approach. Cambridge: Cambridge University Press.

PRITCHETT, L. \& SURYAHADI, A. \& SUMARTO, S. (2000). Quantifying Vulnerability to Poverty: A Proposed Measure, Applied to Indonesia. Washington, DC: World Bank [Working Paper Series, 2437].

REGOLI, A. \& QUINTANO, C. \& CASTELLANO, R. (2003). Income Mobilility in Italy. University of Naples "Parthenope". Institute of Statistics and Mathematics. Hawaii International Conference on Statistics and Related Field, 2003.

RIBAS, R. R. \& MACHADO, A. F. \& GOLGHER, A. B. (2005). "Flutuações e persistência na pobreza: uma análise de decomposição transitória-crônica para o Brasil”. In: Anais XXXIII Encontro Nacional de Economistas, ANPEC.

ROBEYNS, I. (2002). "Sen's capability approach and gender inequality". In: Conference on Promonting Women's Capabilities: examining Nussbaum's Capabilities Approach.

SEN, A. K. (1985a). Commodities and capabilities. Amsterdam. North Holland.

SEN, A. K. (1985b). "The standard of living: lecture I, concepts and critques." In: SEN, A. K. The standard of living: The Tanner lectures. Cambridge: Cambridge University Press. 
SEN, A. K. (1985c). "The standard of living: lecture II, lives and capabilities". In: SEN, A. K. The standard of living: The Tanner lectures. Cambridge: Cambridge University Press.

SEN, A. K. (1992). Inequality Reexamined. Oxford: Clarendon Press.

SEN, A. K. (1999). Development as Freedom. Oxford: Oxford University Press.

SEN, A. K. (1981). Poverty and Famine: An Essay on Entitlement and Deprivation. Oxford: Oxford University Press.

SEN, A. K.; Foster, J. (1997). On Economic Inequality. Oxford: Oxford University Press.

SEN, A. K. (2000). Desenvolvimento como Liberdade. Oxford University Press.

SEN, A. K. (1992). Desigualdade Reexaminada. Clarendon Press, Oxford.

SEN, A. K. (1983). "Development: Which Way Now?” The Economic Journal, vol. 93 p. $745-62$.

SEN, A. K. (2003). Drivers of Escape and Descent: Changing Household Fortunes in Rural Bangladesh. World Development, vol. 31 (3), p. 513-534.

WORLD BANK. (2000). World development report 2000/2001 attacking poverty. Washington, DC: World Bank.

YAQUB, S.(2000). "Intertemporal welfare dynamics: extents and causes". In: Globalization: new opportunities, new vulnerabilities'workshop. Carnegie Endowment.

YAQUB, S. (2003). "Chronic poverty: scrutinizing patterns, correlates and explorations”. Working Paper, n. 21. Manchester: University of Manchester. 
BAGOLIN, I. P.; ÁVILA, R. P.; COMIM, F. V. Pobreza extrema e seus tríplices fundamentos... 